\title{
CpG methylation and various parameters interaction in myotonic dystrophy type I
}

\begin{abstract}
Myotonic dystrophy type 1 is a chronic, slowly progressing, inherited multisystemic autosomal-dominant disease, caused by expansion of CTG repeats in DMPK gene. The purpose of the present study was to analyze molecular expansion profiling of CTG repeat, status of $\mathrm{CpG}$ methylation at $D M P K$ gene locus, and to established relationship between CpG methylation and CTG repeat expansion size along with other clinical and biochemical parameters. Clinically suspected 21 DM1 subjects, 56 family members and 50 normal individuals were included in this study. Molecular diagnosis of CTG repeat expansion was performed by Myotonic Dystrophy Short PCR (MDSP) and Triplet primed-PCR (TP-PCR) and followed fragment analysis on ABI-310 Genetic Analyser. The CpG methylation was done by bisulphite conversion kit (Cells to $\mathrm{CpG}^{\mathrm{TM}}$ Bisulphite conversion kit, 4445555) and 7500 Fast RT-PCR. SPSS version 16 and Pearson correlation coefficient were used for statistical analysis. All clinically suspected 21 subjects had CTG repeat expansion. Among 56 family members, 16 were permutated, and 40 were normal for CTG repeat. Our previous findings (Kumar et al, 2016 and 2018) highlighted that pattern of CTG repeats differs according to ethnicity. Among positive DM1 samples ( $\mathrm{n}=21), 13$ samples were methylated. CpG methylation was significantly correlated only with CTG repeat expansion. This methylation may affect the disease environment and expression of neighbor gene which is responsible for disease pathogenesis.
\end{abstract}

Keywords: myotonic dystrophy type 1 (DM1), CTG repeat expansion, TP-PCR, RTPCR, CpG methylation, bisulphate, myotonin protein kinase, muscular dystrophy, distal muscles
Volume 9 Issue I - 2020

\author{
Ashok Kumar,' Sarita Agarwal,' Sunil \\ Pradhan $^{2}$ \\ 'Department of Genetics, Sanjay Gandhi Post Graduate Institute \\ of Medical Sciences, India \\ 2Department of Neurology, Sanjay Gandhi Post Graduate \\ Institute of Medical Sciences, India
}

\begin{abstract}
Correspondence: Ashok Kumar, Department of Genetics, Sanjay Gandhi Post Graduate Institute of Medical Sciences (SGPGIMS), Lucknow-India-2260 I4, Tel +91-522-2494356, +91745792215I, Email chemistry.ashok83@gmail.com
\end{abstract}

Received: January 25, 2020 | Published: February 26, 2020
Abbreviations: DM1, myotonic dystrophy type 1; UTR, untranslated region; FA, friedreich ataxia; SCA, spinocerebellar ataxia; SCK, serum creatinine kinase; NCV, nerve conduction velocity; SSS, severity of sum score

\section{Introduction}

Myotonic dystrophy type 1 (DM1) is the most common type of adult muscular dystrophy and inherited as autosomal dominant manner. It is characterized by myotonia, wasting of distal muscles, cataracts, cardiac arrhythmias, hypogonadism, frontal balding, and mental impairment etc. ${ }^{1}$ It is caused by an abnormal expansion of the CTG sequence located at the $3^{\prime}$ untranslated region (UTR) of the myotonin protein kinase gene (DMPK) at chromosome $19 \mathrm{q} 13.3 .{ }^{2-4} \mathrm{It}$ varies in normal population from 5 to 35 and beyond 50 repeat is responsible for DM1 severity. ${ }^{5-8}$ DMPK gene is $\sim 14 \mathrm{~kb}$ and encodes $2.3 \mathrm{~kb}$ of mRNA with 15 exons and have 624 amino acids containing serine-threonine kinase protein. ${ }^{9,10}$

There are two precise mechanisms for disease pathogens of DM1 First, the expanded CUG repeats containing RNA expression alters the activity of RNA splicing factors and the consequence is that unregulated alternative splicing of many different genes. ${ }^{11}$ The other mechanism illustrate that the expanded CTG repeats interfere with local chromatin configuration and affects expression of both $D M P K$ and neighboring genes DMAHP (dystrophia myotonica associated home domain protein)/SIX5, dystrophia myotonica containing WD repeat motif (DMWD), and muscle blind protein (MBNL1). ${ }^{12}$ Expanded CTG repeats interferes with the transcription of the adjacent DMAHP gene and thus play a role in disease manifestation. ${ }^{13}$ The decreased Six 5 transcription is important in DM1's pathophysiology as cataract formation..$^{14}$ Additionally, anti-sense strand expression of $D M P K$ also modified by expansion. ${ }^{15}$ Expanded DM1 repeats altered chromatin packaging at DM1 locus as compared to wild-type allele. This DM1 chromatin packaging is mediated by $\mathrm{CpG}$ methylation sensitive binding of the chromatin insulator CTCF proximal to the CTG repeat. Other diseases like fragile $\mathrm{X}$ syndrome (FRAXA), Friedreich ataxia (FRDA) etc. have differential CpG methylation pattern and chromatin packaging which manifest gene expression. ${ }^{16,17}$

Diagnosis of DM1 in clinical setup is difficult, because it contains similar clinical symptoms with spinocerebellar ataxia (SCA) and Friedreich ataxia (FA) and ther triplet repeat disorders. On clinical scale, myotonic dystrophy is diagnosed by the high level of muscle Serum creatinine kinase (SCK), characteristics electromyography (EMG) peaks, nerve conduction velocity (NCV), muscle biopsy, and several other variables. The variability in clinical features of DM1, require molecular testing for accurate diagnosis. Molecular methods used for detection of normal and expanded alleles of disease, are PCR-RFLP (Restriction fragment length polymorphism), ${ }^{18}$ Nested and fluorescent PCR, ${ }^{19,20}$ multiplex PCR and Southern blotting, ${ }^{21-23}$ and Triplet Primed-PCR (TP-PCR), ${ }^{24}$ and a choice methodology has been incorporated. In India, still the diagnosis is totally confined to clinical characterization with no prenatal and counseling set up.

Like various genetic markers, $\mathrm{CpG}$ methylation may also play role in the stability of repetitive sequence, ${ }^{25,26}$ and especially in behaviour of the CTG repeat, ${ }^{27,28}$ and in the pathogenesis of disease. ${ }^{27,29,30}$ 
Therefore, it is necessary to find out the molecular expansion profiling of CTG repeat (by normal and TP-PCR analysis), \& status of methylation on $\mathrm{CpG}$ sites in the myotonic dystrophy locus, and to point out co-relation of $\mathrm{CpG}$ methylation with $\mathrm{CTG}$ repeat expansion size and other clinical and biochemical parameters.

\section{Materials and methods}

\section{Clinical evaluation}

Institutional Ethics Committee of SGPGIMS approved this study. Patients were diagnosed from March 2016 to Dec 2018. Total 21 DM1 patients and 56 related individuals (peripheral blood) from 21 families were referred to SGPGIMS OPD \& IPD and also 50 normal individual were diagnosed for CTG repeat diagnosis. No cell lines were used for the study. Patients having disorder of muscle wasting, jaw and temporal wasting, impairment in gripping capacity, arrhythmia, facial weakness and hypersomnia included in this present study while patients having any other neurological disorder or any other severe or familiar disease excluded from the present study. Clinically, DM1 patients evaluated by detailed disease history, age of onset, physical examination, biochemical testing and electrophysiological testing like NCV (nerve conduction velocity), and EMG (electromyography) and by several other variables. There is certain inclusion and exclusion criteria of the study are as follows:

Inclusion criteria for DM1- Clinical output of DM1, muscle wasting with arrthymia, muscle wasting with facial weakness, and impairment in gripping.

Exclusion criteria- Presence of any other neurological disorder, and presence of strong family history of congenital and severe disease except DM1

\section{Grading of clinical severity}

For evaluation a scoring system was adopted to assess the severity of the salient clinical features and this scale has provided 0 (minimum)-70 (maximum) points. ${ }^{8,31}$ The value 70 highlights severe illness (Table 1). In Indian scenario, clinical score ranging from 8-39 suggesting a mild to moderate severity of disease. The degree of muscle impairment was measured by a five point Muscular Disability Rating Scale (MDRS) measure. ${ }^{32}$

\section{Molecular genetic analysis}

\section{Extraction of DNA samples}

DNA isolation was processed by standard phenol chloroform method. The absorbance ratio at 260 and $280 \mathrm{~nm}$ of DNA was around 1.7-1.9. The quality and purity was assessed by $0.8 \%$ agarose gel electrophoresis in $1 \mathrm{X}$ TBE buffer.

\section{PCR and CTG repeat expansion analysis}

Myotonic Dystrophy Short PCR (MDSP) was used to determine the sizes of normal and/or permutated triplet repeats. PCR was performed in a reaction volume of $25 \mu \mathrm{l}$ using $50 \mathrm{ng}$ genomic DNA with 5pmols of each primers 101-F (5'-FAM-CTT CCC AGG CCT GCA GTT TGC CCA TC-3') and 102-R (5'-GAA CGG GGC TCG AAG GGT CCT TGT AGC-3'). ${ }^{3}$ The cycling profiles were: $5 \mathrm{~min}$ at $95^{\circ} \mathrm{C}, 34$ cycles of $10 \mathrm{sec}$ at $95^{\circ} \mathrm{C}, 30 \mathrm{sec}$ at $62^{\circ} \mathrm{C}$, and $30 \mathrm{sec}$ at $72^{\circ} \mathrm{C}$. A final extension at $72^{\circ} \mathrm{C}$ for $10 \mathrm{~min}$ completed the reaction. Repeat size was calculated by subtracting the number of base pairs of the flanking region from the total length of the PCR products and dividing the result by three. ABI PRISM 310 Genetic Analyzer with the Gene Mapper ID 3.1 software (Applied Biosystems, Foster City, CA, USA) was used for fragment analysis.

For expanded CTG repeat allele expansion, Triplet Repeat PCR (TP-PCR) was carried out in a reaction volume of $25 \mu 1$ using $50 \mathrm{ng}$ genomic DNA with 10 pmol of primer P1-F (5'-FAM-AGA AAG AAA TGG TTC TGT GAT CCC-3'), 8pmol of primer P3-R (5' - TAC GCA TCC CAG TTT GAG ACG-3'), and 2 pmol of primer P4CTG (5' TAC GCA TCC GAG TTT GAG ACG TGC TGC TGC TGC TGC T-3')..$^{24}$ The reaction conditions were as: $10 \mathrm{~min}$ at $96^{\circ} \mathrm{C}, 30$ cycles of 1 min at $94^{\circ} \mathrm{C}, 1 \mathrm{~min}$ at $60^{\circ} \mathrm{C}, 2 \mathrm{~min}$ at $72^{\circ} \mathrm{C}$, followed by final extension at $72^{\circ} \mathrm{C}$ for $10 \mathrm{~min}$. Again, the fragment analysis was done by $\mathrm{ABI}$ PRISM 310 Genetic Analyzer.

\section{Assessment of repeat expansion}

CTG repeat expansion carried out in all individuals $(n=77)$ belonging from $21 \mathrm{DM} 1$ family and also in 50 control subjects. Complete family screening was performed in twelve DM1 families (family 1, 3, 4, 8-11, 13-15, 17, 18).

\section{CpG methylation at myotonic dystrophy locus}

\section{Bisulphite conversion}

Bisulphite conversion kit (Cells to $\mathrm{CpG}^{\mathrm{TM}}$ Bisulphite conversion kit, 4445555) was used for conversion of isolated DNA sample into bisulphite converted DNA as per manufacturer protocol. The PCR reactions were subjected to 2 cycles of $65^{\circ} \mathrm{C}$ for $30 \mathrm{~min}$. and $95^{\circ} \mathrm{C}$ for $1.5 \mathrm{~min}$., and finally $65^{\circ} \mathrm{C}$ for $30 \mathrm{~min}$.

\section{Real Time-PCR (RT-PCR)}

The 7500 Fast RT-PCR was used for $\mathrm{CpG}$ methylation of myotonic dystrophy locus by using following set of primers (F-5'AGAGGGAGTAGTAGTYGTTAGTTTAGTTT3';R5'ACTCCRATTTCCCTATAACCCAAA3' and probes: Meth-[6 FAM]TCCCACCCTAAACGCCGACCTCTCCC[QSY] and Nonmeth[HEX]TCCTCCCACCCTAAACACCAACCTCTCCC[QSY]. The PCR reactions were subjected to 40 cycles of $95^{\circ} \mathrm{C}$ for $10 \mathrm{sec}$, $52^{\circ} \mathrm{C}$ for $30 \mathrm{sec}$., and $72^{\circ} \mathrm{C}$ for $1 \mathrm{~min}$. followed by a $30 \mathrm{sec}$. extension at $72^{\circ} \mathrm{C}$.

\section{Statistical analysis}

Appropriate statistical analysis software (Student $\mathrm{t}$ test and Pearson/Spearman correlation) and SPSS (version 16) were used for analysis. A $p$ value of $<0.05$ was statistically significant.

\section{Results}

\section{Clinical and biochemical analysis}

During the period of about three year (March 2016 to Dec 2018), blood samples $(n=77)$ from 21 DM1 families had been collected at SGPGIMS. Among them 21 had pathogenic DM1, corresponds to $27.27 \%$ of total individual. Mean age at onset of symptoms, mean age of presentation (sampling age), mean duration of illness were $23.86 \pm 9.86$ (range 1-38 year), $27.76 \pm 9.74$ (range $8-40$ year), $3.90 \pm 1.55$ (range 2-7 year) respectively. Similarly, the no. of functionally independent, minimal help, dependent for daily activities, and wheel chair bounds patients were $5,12,3,1$ respectively (Table 1 ). 
Table I Clinical features and severity scale and scores in 21 DMI patients

\begin{tabular}{|c|c|c|c|c|c|c|c|c|c|c|c|c|c|c|c|c|c|c|}
\hline $\begin{array}{l}\text { Patient/ } \\
\text { Sex }\end{array}$ & $\begin{array}{l}\text { Age } \\
\text { at } \\
\text { onset } \\
\text { (y) }\end{array}$ & $\begin{array}{l}\text { Age at } \\
\text { presentation } \\
\text { (y) }\end{array}$ & Dur(y) & FH & MT & WS & WK & DM & $C D$ & D & ADL & Asp & $\mathbf{T}$ & CK & NC & EMG & Score & MDRS \\
\hline & & & & & 7 & 6 & 15 & 3 & 6 & 6 & 6 & 6 & 6 & 6 & 1 & 2 & 70 & \\
\hline I/F & 15 & 17 & 2 & $\mathrm{n}$ & 3 & 4 & 8 & - & - & - & 2 & - & - & 1 & 1 & 2 & 21 & 2 \\
\hline $2 / M$ & 11 & 14 & 3 & $\mathrm{P}$ & 3 & 5 & 12 & - & - & 2 & - & - & - & 1 & 1 & 1 & 25 & 3 \\
\hline $3 / M$ & 36 & 40 & 2 & $\mathrm{P}$ & 4 & 4 & 6 & - & - & - & - & 2 & 4 & - & 1 & 1 & 22 & 4 \\
\hline $4 / M$ & 18 & 20 & 2 & $\mathrm{~m}$ & 4 & 6 & 7 & - & - & - & 2 & - & - & - & 1 & 1 & 21 & 3 \\
\hline $5 / M$ & 20 & 22 & 2 & $\mathrm{n}$ & 4 & 4 & 6 & I & - & 2 & - & - & - & - & 1 & - & 18 & 2 \\
\hline $6 / M$ & 25 & 30 & 5 & $\mathrm{P}$ & 4 & 4 & 8 & - & - & - & - & - & 2 & 1 & 1 & 1 & 21 & 2 \\
\hline $7 / M$ & 29 & 32 & 3 & $\mathrm{~m}$ & 4 & 5 & 7 & - & - & - & 2 & 4 & - & 1 & - & 2 & 25 & 4 \\
\hline $8 / M$ & 36 & 41 & 5 & $\mathrm{~m}$ & 3 & 5 & 1 & 1 & - & - & 2 & - & - & 1 & 1 & 1 & 15 & 1 \\
\hline $9 / M$ & 23 & 26 & 3 & $\mathrm{~m}$ & 2 & 2 & 4 & - & - & - & 2 & - & 6 & - & 1 & 2 & 19 & 2 \\
\hline $10 / M$ & 38 & 40 & 2 & $\mathrm{~m}$ & 3 & 2 & 4 & - & - & - & 4 & - & - & - & - & 1 & 14 & 3 \\
\hline$I I / M$ & 25 & 28 & 3 & $\mathrm{~m}$ & 4 & 5 & 6 & - & - & 2 & 2 & 2 & - & 1 & 1 & - & 23 & 2 \\
\hline $12 / \mathrm{M}$ & 21 & 25 & 4 & $P$ & 4 & 4 & 7 & 2 & - & - & 2 & 4 & - & I & 1 & 2 & 27 & 3 \\
\hline $13 / M$ & 28 & 31 & 3 & P & 7 & 6 & 8 & - & - & - & - & - & 4 & - & - & 1 & 26 & 3 \\
\hline I4/M & 13 & 18 & 5 & $\mathrm{~m}$ & 4 & 5 & 6 & 2 & 4 & - & 4 & 2 & - & - & 1 & 1 & 29 & 5 \\
\hline $15 / M$ & 10 & 15 & 5 & $n$ & 4 & 4 & 8 & - & 2 & - & 2 & 2 & 6 & 1 & 1 & 2 & 32 & 2 \\
\hline $16 / M$ & 26 & 32 & 6 & $\mathrm{~m}$ & 2 & 5 & 2 & 1 & 4 & 4 & 6 & - & - & 1 & 1 & 1 & 27 & 1 \\
\hline I7/M & 31 & 38 & 7 & P & 3 & 4 & 4 & 1 & 2 & 2 & 2 & - & - & - & 1 & 2 & 21 & 3 \\
\hline I8/M & 30 & 34 & 4 & $\mathrm{~m}$ & 3 & 5 & 3 & - & - & - & 2 & - & 4 & - & 1 & 1 & 19 & 2 \\
\hline $19 / F$ & 36 & 40 & 4 & $n$ & 5 & 5 & 7 & - & - & 2 & 4 & 2 & - & 1 & 1 & 2 & 29 & 3 \\
\hline $20 / M$ & 29 & 32 & 3 & $P$ & 4 & 4 & 1 & - & 2 & 2 & 2 & 2 & - & 3 & 1 & 1 & 22 & 1 \\
\hline $21 / F$ & 1 & 8 & 7 & $n$ & 4 & 5 & 6 & - & - & - & 2 & - & - & I & 1 & 1 & 20 & 2 \\
\hline
\end{tabular}

F, Female; M, Male; Dur, Duration of the disease; FH, Family history; n, Unknown; p, paternal; m, maternal; MT, Myotonia; WS, Wasting; WK, Weakness; DM, Diabetes Mellitus; CD, Cardiac Involvement; D, Dysphasia;ADL, Activities of Daily living;Asp., Aspiration; T,Testicular Atrophy; CK, Creatinine Kinase; NC, Nerve Conduction; EMG; Electromyography; MDRS, Muscular Disability Rating Scale. The maximum score (70) of all parameters were shown

Investigations highlighted normal CK (below $192 \mathrm{U} / \mathrm{L}$ ), between $192-500 \mathrm{U} / \mathrm{L}$, and over $1000 \mathrm{U} / \mathrm{L}$ in $12,11,1$ patients respectively. Nerve conduction was abnormal in $18(85.71 \%)$ and myopathic EMG limited to distal muscles in $12(57.1 \%)$ and to proximal muscle in addition to distal muscle in 7 patients $(33.3 \%)$. The clinical score or severity of sum score (SSS) had varied from 14-32. The 6 patients having a score between 14-20, 14 between 21-30, and 1 between 3132 (Table 1)

\section{Molecular genetic analysis}

Total 21 patients and 56 related individuals were recruited in the present study for molecular diagnosis of the disease by MDSP and TP-PCR methodology $y^{3,8,17}$ (Figure $1 \& 2$ ).

MDSP amplify normal and permutated allele only (Figure 1). TPPCR provide specific amplification pattern for normal, permutated and expanded CTG repeat allele (Figure 2). Out of 21 patients; nine, seven, and five had acquired maternal, paternal and unknown mode of transmission of disease respectively (Table 1). Among 56 family members, sixteen were permutated $(28.57 \%)$, and remaining $(n=40)$ were normal $(71.43 \%)$ for CTG repeat (Table 2). Total twelve families (family 1, 3, 4, 8-11, 13-15, 17, 18) undergo complete family screening. 


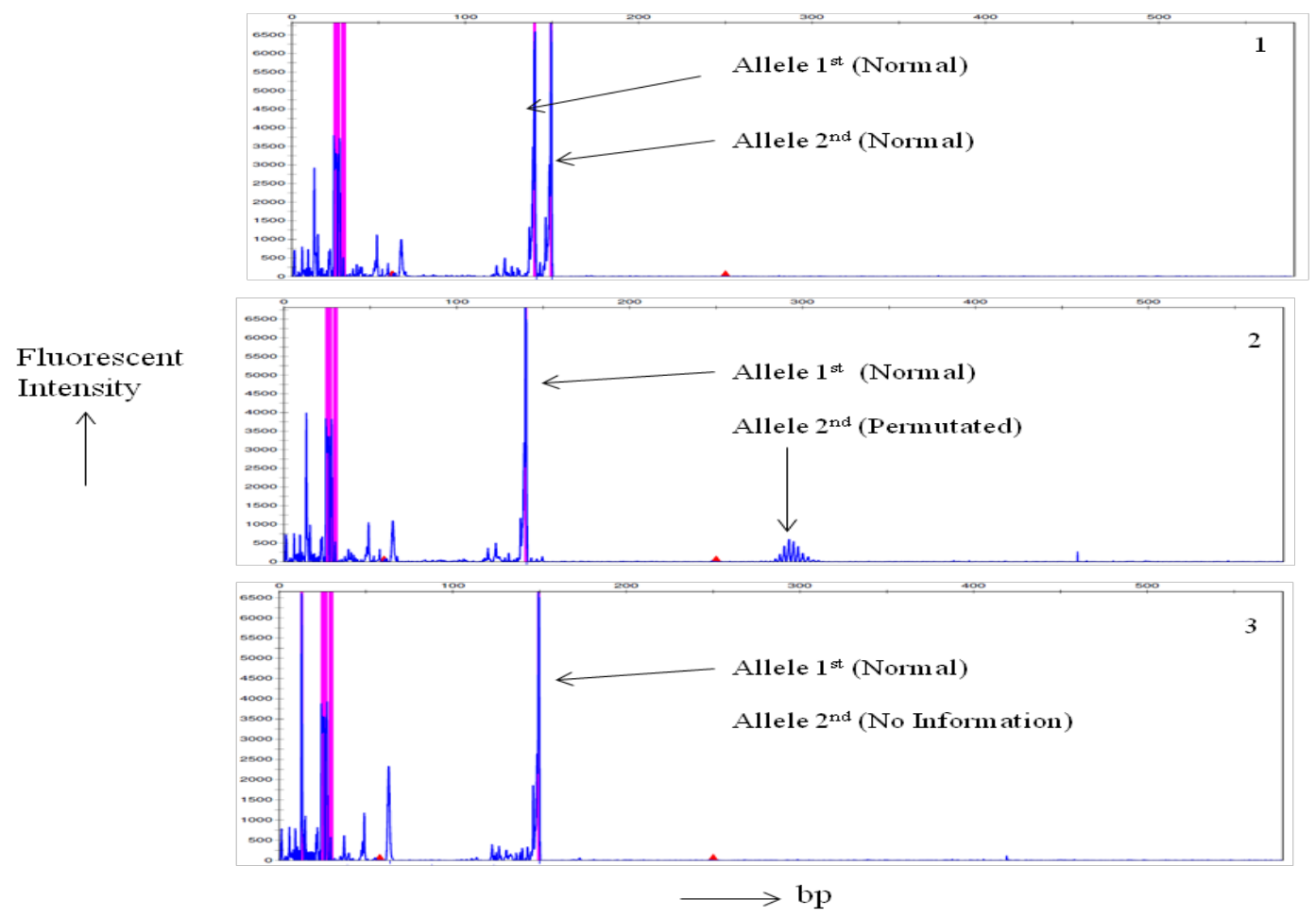

Figure I Myotonic Dystrophy Short-PCR (MDSP) product Gene scan analysis of (I) electrophorogram of a normal individual of a DMI family member (2) an asymptomatic DMI's individual electrophorogram (3) pics of a DMI patient. Horizontal scale indicate size in base pairs while fluorescent intensity of the normal and/or normal/permutated alleles represented by vertical scale.

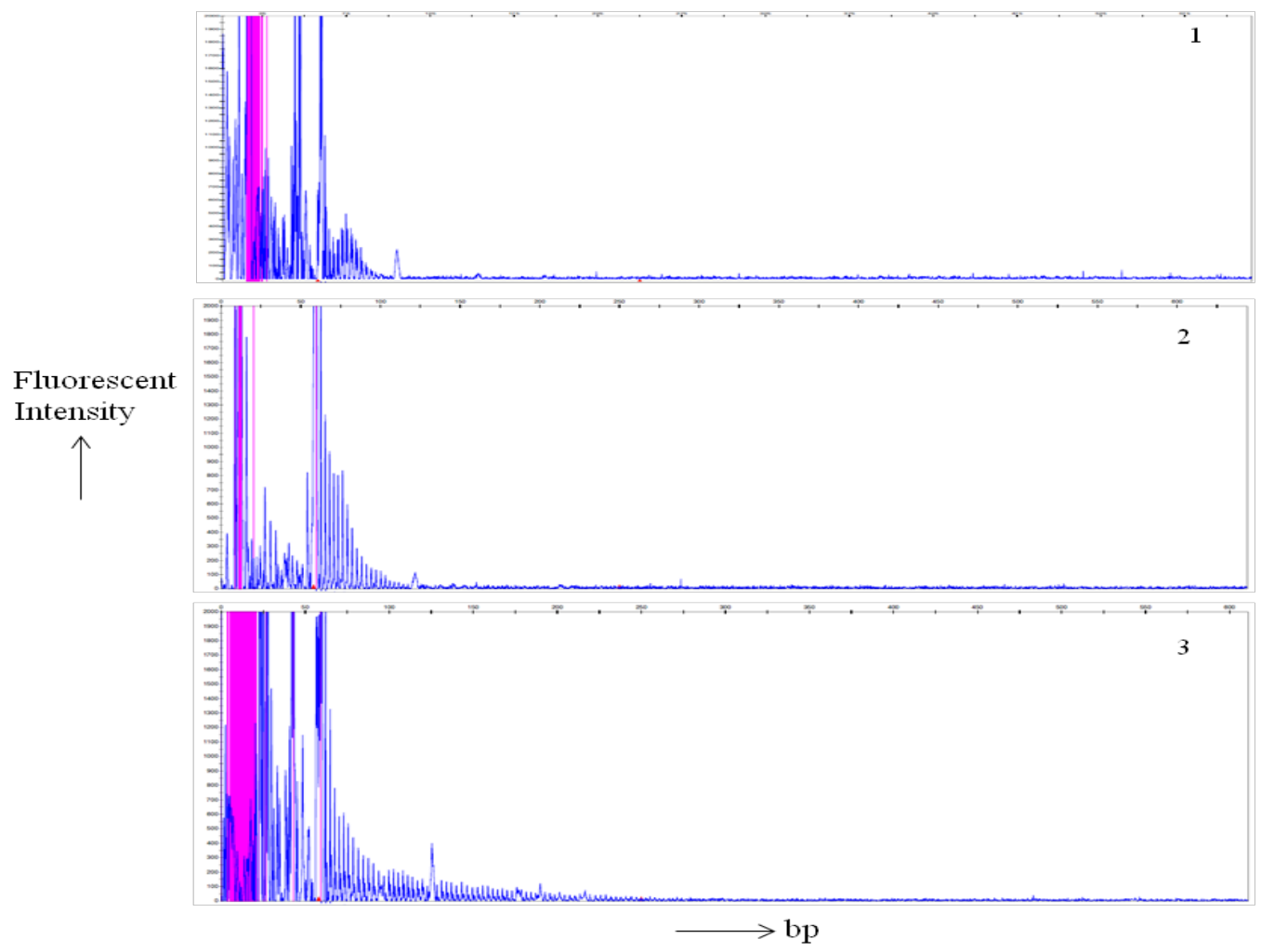

Figure 2 TP-PCR product Gene scan analysis of (I) electrophorogram of a normal individual of a DMI family member (2) an asymptomatic DMI's individual electrophorogram (3) pics of a DMI patient. Horizontal and vertical scales indicate size in base pairs and fluorescent intensity of the expanded alleles respectively. 
Table 2 Molecular profiling status of 2 I DMI families $(n=77)$

\begin{tabular}{|c|c|c|c|c|}
\hline DMI Family(S.N.) & Samples & $\begin{array}{l}\text { Normal PCR CTG repeats } \\
\text { for allele I/allele } 2\end{array}$ & TP-PCR Results & Conclusion \\
\hline \multirow[t]{4}{*}{ Family I } & Patient & $5 / n d$ & Expansion & Full mutation \\
\hline & Patient's Mother & I I-May & Normal & Normal repeat \\
\hline & Patient's Brother & I6-May & Normal & Normal repeat \\
\hline & Patient's Sister & I6-May & Normal & Normal repeat \\
\hline \multirow[t]{2}{*}{ Family 2} & Patient & $3 / n d$ & Expansion & Full mutation \\
\hline & Patient's Father & $16 / p m$ & Confirmed pre mutation & Pre mutated allele \\
\hline \multirow[t]{4}{*}{ Family 3} & Patient & $16 / \mathrm{nd}$ & Expansion & Full mutation \\
\hline & Patient's Father & $16 / p m$ & $\begin{array}{l}\text { Confirmed pre mutation } \\
\text { Normal }\end{array}$ & Pre mutated allele \\
\hline & Patient's Mother & I5-Nov & Normal & Normal repeat \\
\hline & Patient's Brother & II-Mar & & Normal repeat \\
\hline \multirow[t]{5}{*}{ Family 4} & Patient & $\mathrm{II} / \mathrm{nd}$ & Expansion & Full mutation \\
\hline & Patient's Father & I6-Mar & Normal & Normal repeat \\
\hline & Patient's Mother & $\mathrm{II} / \mathrm{pm}$ & Confirmed permutation & Pre mutated allele \\
\hline & Patient's Sister & II-Mar & Normal & Normal repeat \\
\hline & Patient's Brother & II-Aug & Normal & Normal repeat \\
\hline \multirow[t]{3}{*}{ Family 5} & Patient & $16 / n d$ & Expansion & Full mutation \\
\hline & Patient's Father & 16-May & Normal & Normal repeat \\
\hline & Patient's Sister & 16-Mar & Normal & Normal repeat \\
\hline \multirow[t]{3}{*}{ Family 6} & Patient & $16 / \mathrm{nd}$ & Expansion & Full mutation \\
\hline & Patient's Father & I6/pm & Confirmed permutation & Pre mutated allele \\
\hline & Patient's Mother & I5-May & Normal & Normal repeat \\
\hline \multirow[t]{3}{*}{ Family 7} & Patient & $18 / \mathrm{nd}$ & Expansion & Full mutation \\
\hline & Patient's Father & II-Mar & Normal & Normal repeat \\
\hline & Patient's Mother & 10/pm & Confirmed pre mutation & Pre mutated allele \\
\hline \multirow[t]{4}{*}{ Family 8} & Patient & $16 / n d$ & Expansion & Full mutation \\
\hline & $\begin{array}{l}\text { Patient's Mother } \\
\text { Patient's Brother }\end{array}$ & $16 / p m$ & $\begin{array}{l}\text { Confirmed pre mutation } \\
\text { Normal }\end{array}$ & $\begin{array}{l}\text { Pre mutated allele } \\
\text { Normal repeat }\end{array}$ \\
\hline & Patient's Sister & I0-Mar & Normal & Normal repeat \\
\hline & & I5-Nov & & \\
\hline \multirow[t]{3}{*}{ Family 9} & Patient & $3 / n d$ & Expansion & Full mutation \\
\hline & Patient's Father & I5-Sep & Normal & Normal repeat \\
\hline & Patient's Mother & 3/pm & Confirmed pre mutation & Pre mutated allele \\
\hline \multirow[t]{6}{*}{ Family 10} & Patient & $5 / n d$ & Expansion & Full mutation \\
\hline & Patient's Father & 19-Sep & Normal & Normal repeat \\
\hline & $\begin{array}{l}\text { Patient's Mother } \\
\text { Patient's Brother Ist }\end{array}$ & 5/pm & $\begin{array}{l}\text { Confirmed pre mutation } \\
\text { Normal }\end{array}$ & $\begin{array}{l}\text { Pre mutated allele } \\
\text { Normal repeat }\end{array}$ \\
\hline & Patient's Brother2nd & 9-May & Normal & Normal repeat \\
\hline & Patient's Sister & II-Sep & Normal & Normal repeat \\
\hline & & II-May & & \\
\hline
\end{tabular}


Table Continued...

\begin{tabular}{|c|c|c|c|c|}
\hline DMI Family(S.N.) & Samples & $\begin{array}{l}\text { Normal PCR CTG repeats } \\
\text { for allele I/allele } 2\end{array}$ & TP-PCR Results & Conclusion \\
\hline \multirow[t]{5}{*}{ Family II } & Patient & $3 / \mathrm{nd}$ & Expansion & Full mutation \\
\hline & Patient's Father & II-Sep & Normal & Normal repeat \\
\hline & $\begin{array}{l}\text { Patient's Mother } \\
\text { Patient's Brother }\end{array}$ & 3/pm & Confirmed pre mutation & Pre mutated allele \\
\hline & Patient's Sister & I2-Sep & Normal & Normal repeat \\
\hline & & II-Mar & Normal & Normal repeat \\
\hline \multirow[t]{3}{*}{ Family 12} & Patient & $9 / n d$ & Expansion & Full mutation \\
\hline & Patient's Father & 9/pm & Confirmed pre mutation & Pre mutated allele \\
\hline & Patient's Mother & 15-Mar & Normal & Normal repeat \\
\hline \multirow[t]{5}{*}{ Family I3 } & Patient & $9 / n d$ & Expansion & Full mutation \\
\hline & Patient's Father & 9/pm & Confirmed pre mutation & Pre mutated allele \\
\hline & Patient's Brother Ist & II-Mar & Normal & Normal repeat \\
\hline & Patient's Brother2nd & II-Sep & Normal & Normal repeat \\
\hline & Patient's Sister & 16-Mar & Normal & Normal repeat \\
\hline \multirow[t]{4}{*}{ Family I4 } & Patient & $5 / n d$ & Expansion & Full mutation \\
\hline & $\begin{array}{l}\text { Patient's Mother } \\
\text { Patient's Brother }\end{array}$ & $5 / \mathrm{pm}$ & Confirmed permutation & Premutated allele \\
\hline & Patient's Sister & I2-Jun & Normal & Normal repeat \\
\hline & & I5-Mar & Normal & Normal repeat \\
\hline \multirow[t]{3}{*}{ Family I5 } & Patient & $9 / \mathrm{nd}$ & Expansion & $\begin{array}{l}\text { Full mutation } \\
\text { Normal repeat }\end{array}$ \\
\hline & Patient's Father & I0-Mar & Normal & Normal repeat \\
\hline & Patient's Sister & I5-Sep & Normal & \\
\hline \multirow[t]{4}{*}{ Family 16} & Patient & $9 / n d$ & Expansion & Full mutation \\
\hline & Patient's Mother & 9/pm & Confirmed permutation & Premutation allele \\
\hline & Patient's Sister & I8-Dec & Normal & Normal repeat \\
\hline & Patient's Brother & II-May & Normal & Normal repeat \\
\hline \multirow[t]{7}{*}{ Family 17} & Patient & $3 / n d$ & Expansion & Full mutation \\
\hline & Patient's Father & $3 / p m$ & $\begin{array}{l}\text { Confirmed pre mutation } \\
\text { Normal }\end{array}$ & Premutation allele \\
\hline & $\begin{array}{l}\text { Patient's Mother } \\
\text { Patient's Brother Ist }\end{array}$ & 9-May & Normal & Normal repeat \\
\hline & Patient's Brother2nd & 20-Sep & Normal & Normal repeat \\
\hline & Patient's Sister Ist & II-Sep & Normal & Normal repeat \\
\hline & Patient's Sister2nd & I5-Mar & Normal & Normal repeat \\
\hline & & 16-May & & Normal repeat \\
\hline \multirow[t]{4}{*}{ Family 18} & Patient & $\mathrm{II} / \mathrm{nd}$ & Expansion & Full mutation \\
\hline & Patient's Father & II-Mar & Normal & Normal repeat \\
\hline & Patient's Mother & I I/pm & $\begin{array}{l}\text { Confirmed pre mutation } \\
\text { Normal }\end{array}$ & Premutation allele \\
\hline & Patient's Sister & I8-May & & Normal repeat \\
\hline
\end{tabular}


Table Continued...

\begin{tabular}{lllll}
\hline DMI Family(S.N.) & Samples & $\begin{array}{l}\text { Normal PCR CTG repeats } \\
\text { for allele I/allele 2 }\end{array}$ & TP-PCR Results & Conclusion \\
\hline Family 19 & Patient & $9 / \mathrm{nd}$ & Expansion & Full mutation \\
Family 20 & Patient & $10 / \mathrm{nd}$ & $\begin{array}{l}\text { Expansion } \\
\text { Confirmed pre mutation }\end{array}$ & Full mutation \\
& Patient's Father & $10 / \mathrm{pm}$ & Normal & Premutation allele \\
& Patient's Mother & $15-$ May & & Normal repeat \\
Family 21 & Patient & $5 / \mathrm{nd}$ & Expansion & Full mutation \\
\hline
\end{tabular}

nd, Not detectable; pm, Premutation

\section{CpG methylation of DNA samples}

CpG methylation had been done in all DM1 (molecularly confirm) and control samples. The DNA samples containing large CTG repeat expansion were methylated (Table 3) CpG neighbor to DMPK gene locus. Among, 21 positive samples, 13 samples were methylated and rest 8 (DM1 samples) \& 50 (control samples) were unmethylated (Figure 3).
Correlation between $\mathrm{CpG}$ methylation with $\mathrm{CTG}$ repeat expansion, various clinical and biochemical parameters: $\mathrm{CpG}$ methylation was significantly correlated with CTG repeat expansion $(\mathrm{r}=0.679, \mathrm{p}<0.001)$. However, it was not correlated with age at onset, hypersomina, aspiration, dysphasia, dyspepsia, and other parameters (Table 4).

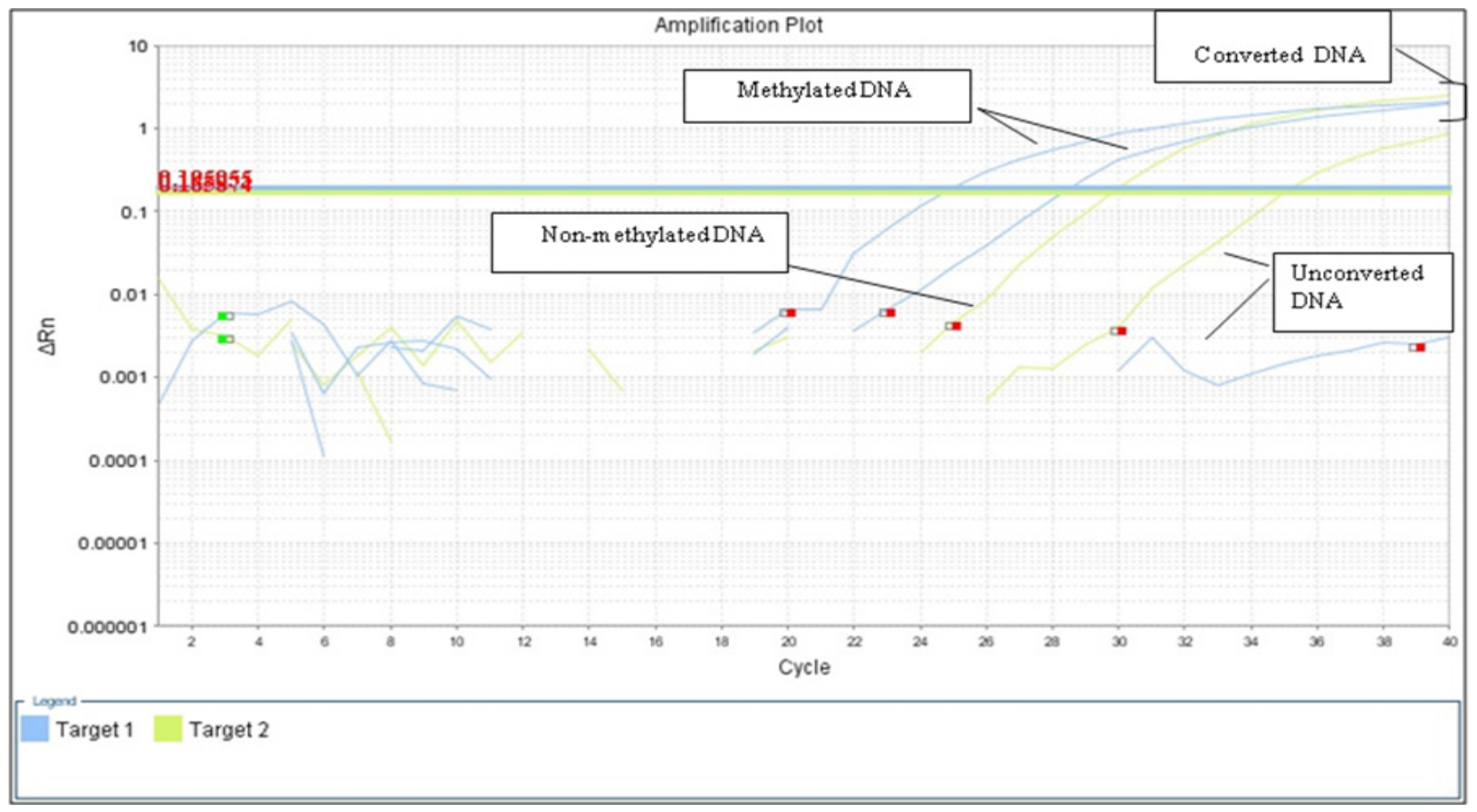

Figure 3 CpG methylation amplification plots of myotonic dystrophy and control samples. Blue (Methylated DNA), green (Non-Methylated), and clump lines show the converted DNA.

Table 3 Methylation status of the studied patients $(n=21)$

\begin{tabular}{llll}
\hline S.No. & Disease status, mutation origin & CTG repeat expansion & Status of CpG methylation \\
\hline I & DMI,Unknown & Large CTG expansion & Methylated \\
2 & DMI,Paternal & Small CTG expansion & Unmethylated \\
3 & DMI, Paternal & Large CTG expansion & Methylated \\
4 & DMI,Maternal & Large CTG expansion & Methylated \\
5 & DMI, Unknown & Small CTG expansion & Unmethylated \\
6 & DMI, Paternal & Small CTG expansion & Unmethylated \\
\hline
\end{tabular}


Table Continued...

\begin{tabular}{|c|c|c|c|}
\hline S.No. & Disease status, mutation origin & CTG repeat expansion & Status of CpG methylation \\
\hline 7 & DMI, Maternal & Small CTG expansion & Unmethylated \\
\hline 8 & DMI, Maternal & Large CTG expansion & Methylated \\
\hline 9 & DMI, Maternal & Large CTG expansion & Methylated \\
\hline 10 & DMI, Maternal & Large CTG expansion & Methylated \\
\hline II & DMI, Maternal & Large CTG expansion & Methylated \\
\hline 12 & DMI, Paternal & Small CTG expansion & Unmethylated \\
\hline 13 & DMI, Paternal & Large CTG expansion & Methylated \\
\hline 14 & DMI, Maternal & Large CTG expansion & Methylated \\
\hline 15 & DMI, Unknown & Large CTG expansion & Methylated \\
\hline 16 & DMI, Maternal & Small CTG expansion & Unmethylated \\
\hline 17 & DMI, Paternal & Large CTG expansion & Methylated \\
\hline 18 & DMI, Maternal & Small CTG expansion & Unmethylated \\
\hline 19 & DMI, Unknown & Small CTG expansion & Unmethylated \\
\hline 20 & DMI, Paternal & Large CTG expansion & Methylated \\
\hline 21 & DMI, Unknown & Large CTG expansion & Methylated \\
\hline
\end{tabular}

Table 4 Correlation between CpG methylation and different parameters

\begin{tabular}{llll}
\hline Variables & CpG methylation r(p-value) & Variables & CpG methylation r(p-value) \\
\hline Age at onset & $0.022(0.925)$ & ADL & $-0.38 I(0.089)$ \\
Age at presentation & $-0.159(0.49 I)$ & Aspiration & $0.139(0.549)$ \\
Duration & $-0.139(0.549)$ & LWD & $-0.277(0.224)$ \\
Facial weakness & $0.285(0.210)$ & CTG repeat expansion & $0.679(<0.001)$ \\
Dyspepsia & $0.010(0.967)$ & CK & $0.552(0.069)$ \\
Dysphasia & $0.347(0.124)$ & Clinical Score & $0.062(0.789)$ \\
Hypersomnia & $-0.062(0.789)$ & MDRS & $0.037(0.872)$
\end{tabular}

LWD, Learning and writing disability

\section{Discussion}

DM1 is a multisystemic autosomal dominant disorder and having phenomena of anticipation. The disabilities are substantial and therefore early detection is mandatory for reproductive counseling of families in which the DM1 has been observed as they have $50 \%$ chances of each pregnancy to have an affected child. The commonly used detection methods utilizes the combination of conventional PCR and triplet repeat-primed PCR. ${ }^{24,33-36}$ The TP-PCR can detect the presence of long allele size without determining the total size of the expansion or the exact number of expanded CTG. Western countries routinely used TP-PCR while in India DM1 is mostly under clinical set up.

The MDSP-TPPCR method used as a convenient tool for the molecular diagnosis of DM1 in this study. The expansions were detected in all 21 patients while among 56 family members, except sixteen (permutated), 40 were normal. Apart from that, 50 normal individuals were subjected to molecular diagnosis of CTG repeat.
A Correlation was observed between the incidence of disease and presence of CTG repeat alleles greater than 19 repeats in the normal population. ${ }^{31,37-39}$ The frequency of alleles with $>19$ CTG repeats differs according to ethnicity., ${ }^{7,40}$ The most frequent allele was different between Caucasians and Asians. Five repeats is the most common in European, and 12 repeats in Japanese and Koreans each. ${ }^{31,41,42}$ In our previous study, (in concordance with other studies), ${ }^{31,37,43,44}$ the frequency of $\mathrm{CTG}$ repeat $>19$ repeats was less than $6 \%$ in normal individuals and $2 \%$ in DM1 families normal individuals. ${ }^{8}$ Similarly, our 2018 study, ${ }^{8}$ highlighted the possible genotype-phenotype correlation between CTG repeat allele frequencies with different clinical, biochemical, molecular parameters and finding provide a clue about association of parameters with CTG repeat frequency.

In the present study, methylation status was studied in case (molecularly confirm)-control study and by using appropriate statistical analysis possible correlation had been observed between CpG methylation with CTG repeat expansion, clinical, biochemical, and laboratory findings. $\mathrm{CpG}$ methylation performed in all $21 \mathrm{DM} 1$ 
(molecularly confirm) and control samples by use of bisulphate treatment and RT-PCR techniques. Among 21 positive samples, 13 samples were methylated and remaining 8 (DM1 samples) \& 50 (control samples) were unmethylated (Figure 3).

Our result highlighted that $\mathrm{CpG}$ sites adjacent to the CTG repeat expansion (large) were methylated, and this methylation was significantly correlated with CTG repeat expansion. However, it was not correlated with age at onset, hypersomina, aspiration, dysphasia, dyspepsia, and other parameters. These findings have same concordance with Shaw et al., ${ }^{29}$ and Steinbach et al. ${ }^{27}$ and contrary to Spits et al., ${ }^{45}$ findings. Similarly in other triplet repeat disorder, level of methylation is positively correlated with the level of length expansion of the fragile X CCG repeat sequence..$^{36}$ Though, the contribution of $\mathrm{CpG}$ methylation to disease pathogenesis is still not fully understood yet methylation might affect the expression of the toxic-CUG DMPK transcript, anti-sense-DMPK expression and/or Six-5 gene. CpG methylation also play an important role in the stability of repetitive sequences, ${ }^{25,26}$ and especially in mode of the CTG repeat \& in the pathogenesis of disease. ${ }^{27,28}$

\section{Conclusion}

In summary, we had successfully established TP-PCR technique in the institute and it is a rapid and non-radioactive technique for detection of CTG repeat expansion in myotonic dystrophy and for other triplet repeat disorder like FRDA, FRAXA, SCA. TP-PCR is a substitute of southern blot (SA) because $\mathrm{SA}$ is radioactive and time consuming methodology. On other hand, status of $\mathrm{CpG}$ methylation was analyzed by bisulphite treatment, RT-PCR techniques. Next, its correlation with CTG repeat expansion, clinical, and biochemical parameters were studied and analyzed. However, more studies should be done in this direction to point out the involvement of $\mathrm{CpG}$ methylation in DM1 pathogenesis.

\section{Acknowledgments}

We are extremely grateful to all patients and their family members for their cooperation in this study and thankful to Sanjay Gandhi Post Graduate institute of Medical Sciences, Lucknow for providing infrastructure facility. Dr. Ashok Kumar is thankful to his Mentor Prof. Sarita Agarwal for time to time encouragement \& guidance, Dr. Sunil Pradhan to providing samples and their clinical evaluation and to DST [NPDF/2015/000951] for providing fellowship.

\section{Conflicts of interest}

The author declares that there are no conflicts of interest.

\section{Funding}

None.

\section{References}

1. Thornton CA. Myotonic Dystrophy. Neurol Clin. 2014;32(3):705-719.

2. Mahadevan M, Tsilfidis C, Sabourin L, et al. Myotonic dystrophy mutation: an unstable CTG repeat in the $3^{\prime}$ untranslated region of the gene. Science. 1992;255(5):1253-1255.

3. Brook JD, McCurrach ME, Harley HG, et al. Molecular basis of myotonic dystrophy: expansion of a trinucleotide (CTG) repeat at the $3^{\prime}$ end of a transcript encoding a protein kinase family member. Cell. 1992;68(4):799-808.
4. Buxton J, Shelbourne P, Davies J, et al. Detection of an unstable fragment of DNA specific to individuals with myotonic dystrophy. Nature. 1992;355(6360):547-548.

5. Kamsteeg EJ, Kress W, Catalli C, et al. Best practice guidelines and recommendations on the molecular diagnosis of myotonic dystrophy types 1 and 2. Eur J Hum Genet. 2012;20(12):1203-1208.

6. Kumar A, Agarwal S, Agarwal D, et al. Myotonic dystrophy type 1 (DM1): A triplet repeat expansion disorder. Gene. 2013;522(2):226-230.

7. Kumar A, Agarwal S, Pradhan S. CTG Repeat Diversity at DMPK Gene Locus in Indian Population. J Investigative Genomics. 2016;3(1):7-13.

8. Kumar A, Agarwal S, Pradhan S. Molecular and clinical spectrum of type 1 myotonic dystrophy. Gene Reports. 2018;11:34-41

9. Mahadevan MS, Amemiya C, Jansen G, et al. Structure and genomic sequence of the myotonic dystrophy (DM kinase) gene. Hum Mol Genet. 1993;2(3):299-304.

10. Shaw DJ, McCurrach M, Rundle SA, et al. Genomic organization and transcriptional units at the myotonic dystrophy locus. Genomics. 1993;18(3):673-679.

11. Ranum LP, Cooper TA. RNA-mediated neuromuscular disorders. Annu Rev Neurosci. 2006;29:259-277.

12. Frisch R, Singleton KR, Moses PA, et al. Effect of triplet repeat expansion on chromatin structure and expression of DMPK and neighboring genes, SIX5 and DMWD, in myotonic dystrophy. Mol Genet Metab. 2001;74(12):281-291.

13. Klesert TR, Otten AD, Tapscott SJ. Trinucleotide repeat expansion at the myotonic dystrophy locus reduces expression of DMAHP. Nat Genet. 1997;16(4):402-406.

14. Klesert TR, Diane H Cho, John I Clark, et al. Mice deficient in Six 5 develop cataracts: implications for myotonic dystrophy. Nat Genet. 2000;25:105-109.

15. Cho DH, Thienes CP, Mahoney SE, et al. Antisense transcription and heterochromatin at the DM1 CTG repeats are constrained by CTCF. Mol Cell. 2005;20(3):483-489.

16. Wang YH. Chromatin structure of repeating CTG/CAG and CGG/CCG sequences in human disease. Front Biosci. 2007;12:4731-4741.

17. Kumari D, Usdin K. Chromatin remodeling in the noncoding repeat expansion diseases. J Biol Chem. 2009;284(12):7413-7417.

18. Shaw DJ, Meredith AL, Sarfarazi M, et al. The apolipoprotein gene C2 gene: subchromosomal localization and linkage to the myotonic dystrophy locus. Hum Genet. 1985;70:271-273.

19. Sermon K, Lissens W, Joris $\mathrm{H}$, et al. Clinical application of preimplantation diagnosis for myotonic dystrophy. Prenat Diagn. 1997;17(10):925-932.

20. Sermon K, De VA, Van de VH, et al. Fluorescent PCR and automated fragment analysis for the clinical application of preimplantation genetic diagnosis (Steinert's disease). Mol Hum Reprod. 1998;4(8):791-796.

21. Sermon K, Seneca S, De Rycke M, et al. PGD in the lab for triplet repeat diseases-myotonic dystrophy, Huntington's disease and fragile- $X$ syndrome. Mol Cell Endocrinol. 2001;183(1):S77-S85.

22. Goossens V, DeRycke M, DeVos A, et al. Diagnostic efficiency, embryonic development and clinical outcome after the biopsy of one or two blastomeres for preimplantation genetic diagnosis. Hum Reprod. 2008;223:481-492.

23. Harton GL, Tsipouras P, Sisson ME, et al. Preimplantation genetic diagnosis for Marfan syndrome. Mol Hum Reprod. 1996;2(9):713-715. 
24. Warner JP, Barron LH, Goudie D, et al. A general method for the detection of large CAG repeat expansions by fluorescent PCR. J Med Genet. 1996;33(12):1022-1026.

25. Gourdon G, Dessen P, Lia AS, et al. Intriguing association between disease associated unstable trinucleotide repeat and $\mathrm{CpG}$ island. Ann Genet. 1997;40(2):73-77.

26. Nichol K, Pearson CE. CpG methylation modifies the genetic stability of cloned repeat sequences. Genome Res. 2002;12(8):1246-1256.

27. Steinbach P, Glaser D, Vogel W, et al. The DMPK gene of severely affected myotonic dystrophy patients is hypermethylated proximal to the largely expanded CTG repeat. Am J Hum Genet. 1998;62(2):278-285.

28. Gorbunova V, Seluanov A, Mittelman D, et al. Genome-wide demethylation destabilizes CTG.CAG trinucleotide repeats in mammalian cells. Hum Mol Genet. 2004;13(23):2979-2989.

29. Shaw DJ, Chaudhary S, Rundle SA, et al. A study of DNA methylation in myotonic dystrophy. J Med Genet. 1993;30(3):189-192.

30. Filippova GN, Thienes CP, Penn BH, et al. CTCF-binding sites flank CTG/CAG repeats and form a methylation-sensitive insulator at the DM1 locus. Nat Genet. 2001;28:335-343.

31. Devi MG, Chaudhary JR, Vasanth A, et al. Correlation of clinical profile of myotonic dystrophy with CTG repeats in the myotonin protein kinase gene. Indian J Med Res. 1998;107:187-196.

32. Mathieu J, De Brackeleer M, Prkvost C, et al. Myotonic dystrophy: clinical assessment of muscular disability in an isolated population with presumed homogenous mutation. Neurology. 1992;42(1):203-208.

33. Kumar A, Agarwal S, Phadke S, et al. Application of a reliable and rapid polymerase chain reaction based method in the diagnosis of myotonic dystrophy type 1 (DM1) in India. Meta Gene. 2014;2:106-113.

34. Radvansky J, Ficek A, Minarik G, et al. Effect of unexpected sequence interruptions to conventional PCR and repeat primed PCR in myotonic dystrophy type 1 testing. Diagn Mol Pathol. 2011;20:48-51.

35. Kumar A, Agarwal S, Pradhan S. Assessment of Premutation in Myotonic Dystrophy Type 1 Affected Family Members by TP-PCR and Genetic Counseling. Case Rep Med. 2014:1-8.
36. Addis M, Serrenti M, Meloni C, et al. Triplet-primed PCR is more sensitive than southern blottinglong PCR for the diagnosis of myotonic dystrophy type 1. Genet Test Mol Biomarkers. 2012;16(12):1428-1431.

37. Basu P, Gangopadhaya PK, Mukherjee SC, et al. Molecular anatomy of CTG expansion in myotonin protein kinase gene among myotonic dystrophy patients from eastern India. Hum Mutat. 2000;16(4):372.

38. Deka R, Majumder PP, Shriver MD, et al. Distribution and evolution of CTG repeats at the myotonin protein kinase gene in human populations. Genome Res. 1996;6(2):142-154.

39. Imbert $\mathrm{G}$, Kretz $\mathrm{C}$, Johnson $\mathrm{K}$, et al. Origin of the expansion mutation in myotonic dystrophy. Nature Genet. 1993;4(1):72-76.

40. Kumar A, Agarwal S, Pradhan S. Variable Ethnic Frequency and Risk Ratio of DMPK gene: A Meta-Analysis Survey. J Steroids Horm Sci. 2015;6(3):160.

41. Martorell L, Monckton DG, Sanchez A, et al. Frequency and stability of the myotonic dystrophy type 1 premutation. Neurology. 2001;56(3):328335 .

42. Choi BO, SunWoo IN, Kim SM, et al. Clinical significance of CTG repeat expansion in Korean myotonic dystrophy patients. J Korean Neurol Assoc. 1999; 17:548-553.

43. Kim SY, Kim JY, Kim GP, et al. Molecular and Clinical Characteristics of Myotonic Dystrophy Type 1 in Koreans. Korean $J$ Lab Med. 2008;28(6):483-492.

44. Pan H, Lin HM, Ku WY, et al. Haplotype analysis of the myotonic dystrophy type 1 (DM1) locus in Taiwan: implications for low prevalence and founder mutations of Taiwanese myotonic dystrophy type 1. Eur J Hum Genet. 2001;9(8):638-641.

45. Spits $\mathrm{C}$, Seneca S, Hilven $\mathrm{P}$, et al. Methylation of the $\mathrm{CpG}$ sites in the myotonic dystrophy locus does not correlate with CTG expansion size or with the congenital form of the disease. J Med Genet. 2010;47(10):700703. 